\title{
Putting pressure on WOMBAT - outcomes and unique capabilities
}

Helen Elizabeth Maynard-Casely ${ }^{1}$, Stanley Lee ${ }^{1}$, Norman Booth ${ }^{1}$, Andrew Studer ${ }^{1}$, Vanessa Peterson ${ }^{1}$, Samuel Duyker ${ }^{2}$, Kazuki Komatsu $^{3}$, Ryo Yamane ${ }^{3}$, Gabriel Murphy ${ }^{2}$, Thomas Vogt ${ }^{4}$

${ }^{1}$ Australian Nuclear Science And Technology Organisation, Kirrawee, Australia, ${ }^{2}$ Department of Chemistry, University of Sydney, Sydney, Australia, ${ }^{3}$ Geochemical Research Centre, University of Tokyo, Toyko, Japan, ${ }^{4}$ University of South Carolina, Colombia, United States

E-mail: helenmc@ansto.gov.au

The high-intensity neutron diffraction instrument at the OPAL reactor (known as WOMBAT) is a highly versatile instrument [1]. The high performance of the instrument comes from the combination of the best area detector ever constructed for neutron diffraction with the largest beam guide yet put into any research reactor and a correspondingly large crystal monochromator, all combine to provide an instrument which is unique in its capabilities within the Southern hemisphere.

There has been a recent increase in our capacity to run high-pressure experiments at the instrument, which has already yielded some fantastic results (for example, [2]). We wish to highlight these outcomes and to present the unique highpressure capability of our facilities; the study of radioactive samples under pressure and the ability to apply high-voltage across samples under high pressure [3].

[1] A. Studer et al. (2006) Physica B: Condensed Matter, 385-386, 1013-1015.

[2] S. Duyker et al. (2016) Nature Chemistry, 8, 270-275.

[3] N. Booth et al. (In review) Journal of Neutron Research.

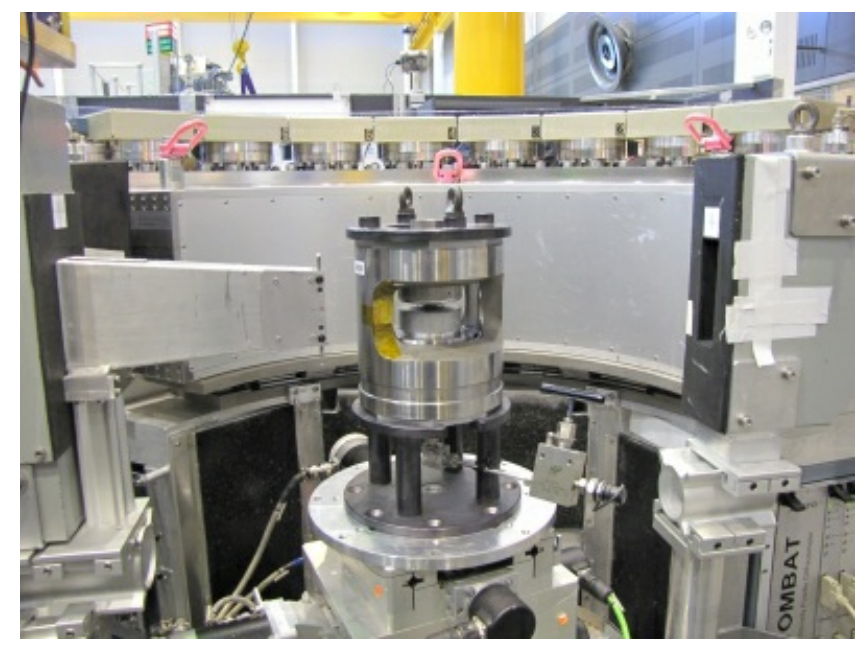

Keywords: Instrument, neutron, high-pressure 